This is the peer reviewed version of the following article: Cummins A.M., Denney-Wilson E. \& Homer C.S.E. (2016) Journal of Nursing Management 24, 614-623. The challenge of employing and managing new graduate midwives in midwifery group practices in hospitals, which has been published in final form at https://

doi.org/10.1111/jonm.12364. This article may be used for non-commercial purposes in accordance with Wiley Terms and Conditions for Self-Archiving. 
Cummins A, Denny-Wilson E, Homer CSE. (2016) The challenge of employing and managing new graduate midwives in midwifery group practices in hospitals. Journal of Nursing Management 24, 614-623.

\section{The challenge of employing and managing new graduate midwives in midwifery group practices in hospitals}

\section{Structured Abstract}

Aim(s)

This study explores the views of midwifery managers and key stakeholders, regarding the facilitators and barriers to employing new graduate midwives in midwifery continuity of care models

\section{Background}

Maternity services in Australia are shifting towards midwifery continuity of care models where midwives work in small group practices, requiring change to the management of staff. Public policy in Australia supports maternity services to be reconfigured in this way. Historically, experienced midwives work in these models, as demand grows; new graduates are employed to staff the models.

\section{Method(s)}

A qualitative descriptive approach exploring the manager's experience of employing new graduate's in the models. Managers, clinical educators and hospital midwifery consultants $(n=15)$ were recruited by purposeful sampling.

\section{Results}

Drivers, enablers, facilitators and barriers to employing new graduates in the models were identified. Visionary leadership enabled the managers to employ new graduates in the models through initial and ongoing support. Managing the myths stemming from fear of employing new graduates to work in midwifery continuity of care models was challenging.

\section{Conclusion}

Managers and other key stakeholders provide initial and ongoing support through orientation and providing a reduced workload.

\section{Implications for Nursing Management}

Visionary leadership can be seen as critical to supporting new graduates into midwifery continuity of care models. The challenges for management to overcome include managing the myths stemming from fear of employing new 
graduates to work in a flexible way around the needs of the women within an organisation culture.

\section{Introduction}

Maternity services in Australia are gradually shifting towards midwifery led continuity of care models and this requires significant changes to the organisation and management of staff. Midwifery continuity of care, also called caseload or midwifery group practice (MGP) is defined as care provided to women throughout pregnancy, birth and the early postnatal period from a single midwife or a small group of midwives (Sandall et al. 2013). Midwifery continuity of care models reduce obstetric interventions such as epidural anaesthesia, episiotomy and caesarean section operations and result in fewer babies being admitted to the neonatal intensive care unit, at no extra cost to the health service (Tracy et al. 2013, McLachlan et al. 2012, Sandall et al. 2013). Consequently, the Australian government has recommended that publicly funded maternity services provide midwifery models of care (Australian Government Department of Health and Ageing 2009, New South Wales Health 2010) although uptake has been slow.

The managers and other key stakeholders in this study employed or supported midwives who provide care to the woman throughout her pregnancy at a time and place that suits the woman and is on call for the birth with most postnatal care provided in the woman's home. Because workload varies according to the needs of the women in their care, traditional models of management are not always suitable for managing midwives working in flexible ways in continuity of care models, and managers need to adapt to managing this different workforce. In Australia, a clinical redesign saw the introduction of 32 fulltime equivalent midwives in one hospital working in a flexible way around the needs of the women, being on-call and focused on women's rather than institutional needs (Hartz et al. 2012). Again, this was a challenge for the structure of the health system and the way these midwives were managed. Midwives in midwifery continuity of care models are employed on an annualised salary, with a certain number of women to care for each year (Hartz et al. 2012). 
Midwifery continuity of care has been found to be a highly satisfying way for midwives to work and is a popular way of working due to the professional relationships that midwives can develop with women and the flexible working arrangements the role enables (Collins et al. 2010, Hartz et al. 2012, Newton et al. 2014). In the past, only experienced midwives have been recruited to work in these models, however as demand grows new graduate midwives are employed to sustain the models of care (Hartz et al. 2012) and this can be challenging for some health services to implement.

\section{Midwifery continuity of care; a manager's challenge?}

Similar to nurses, new graduate midwives have been traditionally employed in some form of "transitional" support from student to registered practitioner for approximately one year. The new graduate year for nurses, known as transitional programs, have become commonplace in many countries (Rush et al. 2015). New graduate midwives, those in their first year post graduation, have not historically worked in continuity of care models despite being prepared to work across the scope of midwifery practice from their degrees (Gray et al. 2012, Cummins et al. 2015). A modification of the transitional program has been adapted for midwives (called a "rotation" year) and sees new graduates working in a number of different settings and rotating every few months through areas such as labour and birth, antenatal ward, antenatal clinic and postnatal wards. Several studies (Panettiere and Cadman 2002, Passant et al. 2003, Kensington 2006, Lennox et al. 2008, Davies 2009, Davis et al. 2011, Hughes and Fraser 2011, Barry 2011) have examined the experience of newly graduated midwives during the transition year, however, there is limited evidence about new graduate midwives who are initially placed within midwifery continuity of care models rather than in this rotational model.

When new graduate midwives work in midwifery continuity of care models they are orientated into a small group of midwives and provide care to a caseload of women. Their practice is flexible in nature, that is, they work their own hours to suit the women's needs providing antenatal care at a time suitable to the woman and the midwife, they are on-call for the birth and then provide postnatal care often visiting the woman at home. New graduate midwives, working in this 
flexible way, are supported by more experienced midwives and mentors via text message, the phone, in person and through regular team meetings (Kensington, 2006; Lennox, 2012; Lennox 2008; Cummins et al 2015).

Our previous work has found that new graduate midwives who work in continuity of care are well supported to develop relationships with the women and the small group of midwives they work alongside (Cummins et al. 2015). The new graduates' consolidated their skills when working with the woman and were supported from within the small group of midwives. Working in a continuity of care model was found to be satisfying while sustaining the model of care for women (Cummins et al. 2015). Although the study was conducted with a small number $(n=13)$ of new graduate midwives it led to the question of why new graduates are not being employed in midwifery continuity of care models.

Following on from our previous work, the following research question was raised as phase two of the study:

What are the experiences of managers and other key stakeholders when they employ new graduate midwives in midwifery continuity of care models?

Phase two of the research aimed to explore the views of midwifery managers, educators and other key stakeholders. The questions asked focused on the facilitators and barriers to employing new graduate midwives in midwifery continuity of care models. This paper reports on the second phase of the research project.

\section{Method}

A qualitative descriptive approach was used to describe and explore the manager's experience and perspective of employing new graduate midwives in midwifery continuity of care models. The exploration was based on the interest in, and understanding of, the "who, what and where" of their experiences (Sandelowski 2000 p338). There is little known on the facilitators and barriers to employing new graduate midwives in continuity of care models therefore a 
qualitative descriptive approach is a useful form of inquiry to describe how the managers feel (Sandelowski 2000).

Ethical approval was sought and granted by the University of Technology Sydney ethics committee. (HREC Approval Number: 2012000328).

\section{Participants}

Fifteen managers, educators, clinical midwifery consultants and clinical support midwives were recruited by purposeful sampling. As our question was to enquire why new graduates were employed or not, we needed to recruit managers to the study however managers are not solely responsible for supporting staff in continuity of care models. The other staff responsible for the support of new graduate midwives are midwifery clinical educators and consultants, and they were recruited as key stakeholders in supporting new graduates as they transition from student to an autonomous practicing midwife (Hartz et.al. 2012). The managers, educators, clinical midwifery consultants were contacted via email for a phone interview. Maternity services that offer midwifery continuity of care to women were identified from phase one of the study and are mostly in metropolitan settings, all are publicly funded. Participants were recruited from the following states in Australia, New South Wales, Australian Capital Territory, Victoria, Queensland and South Australia (Table 1). The states have been de-identified as there are only a small number of maternity services that provide continuity of care in each state and the managers could be identified. Privately practicing midwives providing caseload midwifery were not included in the sample as they were less likely to recruit new graduate midwives.

Insert table 1 here

\section{Data collection}

Phone interviews were the most convenient to both the managers and the researchers as the participants were from all over Australia. The first author was responsible for data collection. Face to face interviews are usually conducted in qualitative research however participants views of telephone 
interviews have been positively reported in other qualitative studies (Holt 2010; Ward, Gott \& Hoare 2015). The telephone is commonly used and participants report the value of anonymity when undertaking interviews as part of qualitative research. Telephone interviews should be considered depending on the nature of the participant group and the proposed methods of data analysis (Holt 2010; Ward, Gott \& Hoare 2015). Midwifery key stakeholders regularly use telephones for meetings and as the interviews are easily recorded with a hand held device with the participants consent, telephone interviews were the first choice of method for data collection. Semi structured interviews were conducted and the interviews were recorded with permission and transcribed verbatim. The participants were asked a series of questions about their decision to employ new graduate midwives to work in midwifery continuity of care, and the questions are included in table 2. The researcher knew some of the participants and there was the potential for the blurring of boundaries (Burns et al 2012). The researcher positioning herself as a researcher rather than a colleague or acquaintance addressed the issue of reflexivity. The other two authors on the research team read de-identified data and therefore were outsiders (Burns et al 2012) to the data collection thus enhancing rigour.

Table 1 Questions

Insert table 2 here

\section{Analysis}

Thematic analysis was undertaken using several phases, the first phase involved the researcher becoming familiar with the data with initial coding of the data, then searching for themes, sub-themes, reviewing the themes and finally the themes were named and defined before writing the report of the findings, as described by Braun and Clarke (2006).

By reading the transcripts, the first author $(A C)$ familiarised herself with the data. The data was read and then reread systematically generating initial codes across the entire data set (Braun \& Clarke 2006). The first stage coding was conducted manually, organising the data into meaningful groups in a table 
(Braun and Clarke 2006). This was the beginning of an audit trail that allowed the other authors to understand how the themes emerged and conclusions were reached (Lavender et al 2004)

The next phase of analysis was to look for themes within the organised data (Braun and Clarke 2006). The initial themes that emerged from the data were: a) facilitating new graduate midwives in the continuity of care models in order to meet staffing needs; and, b) providing support to the new graduates. These key themes have captured important elements from the data in relation to the research question (Braun \& Clarke 2013). Sub themes included support, such as providing a longer orientation period and a reduced caseload for new graduate midwives. Key themes that emerged as barriers to employing new graduate midwives were based on the historical nature of traditional rotation programs and the organisational expectations of new graduate midwives. Under the key themes were sub themes such as organisational culture and fear of "something going wrong". This description of the data was further analysed to discover the who, what and where (Sandelowski 2000) of the managers' and other key stakeholders' experience. The analysis was driven by the data and not by a theoretical framework (Braun and Clarke 2006) in order to understand the experiences of the managers and other key stakeholders. By reflecting and comparing the emergent themes with the other two authors ( $\mathrm{CH}$ \& EDW) further analysis occurred. We drew a concept map that showed where themes overlapped and were able to write a narrative around each theme, this exercise enabled the final naming of the themes.

The first author took responsibility for the phases of the analysis, looking for the points of interest in relation to the topic extracted from the themes and subthemes to arrive at a final interpretation of the data (Braun \& Clarke 2006). The constant checking and rechecking of emerging themes between the authors and the use of verbatim quotes from the participants were all steps to ensure rigour in the analysis (Lavender, Edwards \& Alfirevic 2004)

\section{Findings}


Drivers, enablers, facilitators and barriers to employing new graduate midwives in midwifery continuity of care models were identified and a key theme was that having visionary leadership enabled the employment of new graduate midwives in these models. Figure 1 describes the process that was taking place for the managers and in the health system around new graduates.

\section{Figure 1}

Insert figure 1

\section{Drivers - A need to staff the continuity of care models. "We are recruiting for the future"}

Recruitment of midwives to staff the continuity of care models for the future was the main driver for employing new graduate midwives to work in midwifery continuity of care models, with an overarching theme that if they do not recruit new graduates they miss out in two ways: they won't get the best graduates and they will not be able to staff their services. Two managers said "you have to think about your future"; "you build succession planning" (no2) and "so it is our ultimate plan that we have a first year of practice with each one of the group practices then every 12 months we're growing another group practice" (no 10).

Midwives are predominantly female, and managing a feminised workforce meant the managers had to think about recruiting for the future as midwives working with continuity of care models may take maternity leave. These managers illustrated this by saying, "we do have a dominance of women [midwives] having babies" (no 4) and "we've needed them [new graduates] this year because we have had a lot of people [midwives] having pregnancies" (no7). Recruiting for the future includes replacing retiring midwives in the continuity of care models as noted here, "we had a lot of staff who were planning retirement and a few staff who were planning babies" and then goes onto say "new graduates are a really important part of our recruitment strategy" (no1). 
On the other hand, one key stakeholder who did not employ new graduates indicated that she had heard "we're not coming to you because you don't offer us a caseload as part of our graduate year" (no 9). This key stakeholder was worried that she would not be able to recruit the future midwifery workforce and recognised that change was needed. A clinical midwifery consultant supports this stance by saying "the [new graduate] midwives end up being dissatisfied and they probably leave because they're in that fragmented model of care" (no 5). Recruiting for the future was a clear driver for staffing the continuity of care models.

Having employed new graduates into the continuity of care models has built the workforce capacity, for example, "more than $50 \%$ of our staff now working there started as new graduates" and "75\% have stayed" (no 1). Employing new graduate midwives to the model is important this manager said that "find ways of getting new graduates' in" [into the model] and "working with new graduates is imperative if we are going to grow the profession" (no 2). Hence the need to staff and maintain the midwifery continuity of care models was based on employing new graduate midwives into the model.

Another educator discussed removing barriers in order to staff and sustain the continuity of care models, "if we are going to sustain the model we need to be more accepting to putting up less barriers about how much experience [new graduate experience]" (no 11). Another manager took advice from the midwives working within the caseload practice, "the girls [midwives] saw the sustainability of the model was to actually encourage the recruitment of new graduates" (no 13) and evident here "they (caseload midwives) look around and think who will fit into our service and be good with the women" (no 11). There was a reported fear of losing the models "we are going to be left with no continuity model if we don't do something" (no 1), that is employ new graduates.

\section{Enablers - Preparing students, recruiting new graduates and providing orientation}




\section{"We have been preparing students to work in the continuity of care models".}

An enabler for employing new graduate midwives was the process they had undertaken to prepare midwifery students to work in the models. One manager said, "we are training students to work against the scope of practice why would we not encourage them to work in a continuity model" (no2). Another discussed the point of competence at the time of registration, saying "they come out competent, they come out knowing, many of them have been exposed to continuity of care models" (no4) and again, "new grads come straight from their training, they want to see a woman through a journey, they've already done that with their follow-throughs, I think a lot of them want to continue working in that way" (no12). On the other hand, one manager discussed the new graduates varying degrees of ability as noted here "some universities do prepare students more effectively than others, the product I get at the end as an employer is actually quite different even though they've had to reach a certain standard" (no 9). It is the continuity of care experiences in the midwifery student education that this educator saw as most important, "the continuity is what has prepared the students to work in the continuity models" [students] (no 15).

Managers discussed aligning certain students to work in continuity models, "we ask them what their future plans are and what they want to do with their career and we will sort of like, pick some people to go to various areas we will change their rotation program" as students (no 11). Again, these managers discuss preparing the students to work in continuity "they have had 6-8 weeks in their training with a continuity of care group, then obviously you're hoping that could be part of the succession planning" (no 6) and "we have really good relationships with the midwifery students so we tend to know a lot of them by the time they actually come and do the transition year" (no 7).

The managers discussed having well prepared midwifery students to transition straight into the model, "here we have always had problems getting staff so about 10-12 years ago we purposely set about growing our own workforce there's a number of students with the plan that we may retain at least 50-60 percent of those students" (no 10) and here "we needed staff so I kind of sought 
her out because I knew her - she'd spent a bit of time in the birth centre as student" (no 8).

There were repeated reports that the new graduates' need support "you don't just throw them in, you put support systems there to support them in order to make it successful" (no 11).

\section{"We provide a good orientation"}

The managers who do employ new graduates indicated that the orientation period was the key to transitioning the new graduate into the continuity of care model, "they are really well supported in that first four to six weeks and the workload is gradually increased, they start with a reduced caseload" (no 1). A reduced caseload means the new graduate does not provide care for the same number of women as other midwives who are more experienced. A longer orientation period is described by this manager "give them high level support for the first two or three months, they find their feet and they fly" (no 2). Having to reduce the caseload was not always seen as helpful, "the downside of a reduced caseload is the impact on the workload for the other midwives in the group" (no 6). It is important that the small group of midwives are cohesive as the other midwives in the group support the new graduate to transition from student to autonomous practitioner.

\section{Facilitator - The new graduates need support "Getting support from within the group"}

The managers reported different models of support although new graduate midwives were often supported through a buddy or mentoring system within the small group of midwives they worked alongside. For example, "they have a direct buddy they are in a group of four" (no 14). Again reported here "there are four of them they have a buddy and then they've all got [mobile] phones, so they can talk to each other" (no 8) and "they get a week's orientation, then they work in partnership with the mentor for a while, so for the first few months they work the same clinic (off campus, various points around council but not in people's homes) as their mentors" (no10). Mentoring was, at times, arranged however other managers let the new graduate find their own support "I haven't 
assigned them to a mentor it has been a natural sort of attraction" (no 13) and here "they do buddy up, find a particular person they get on well with and maybe that's not their work partner" (no 12). Other support discussed from within the group of midwives, "they don't have a buddy, they work with a team" (no 15) and "midwives in the team support them" (no 2). Letting the new graduates find their own support aimed to reduce personality differences, reported by one midwifery consultant as a problem, saying "we should not be relying on the group to mentor a new grad in" (no 6). This led to a discussion of other support mechanisms including a support plan for the new graduate to consolidate their skill base.

\section{Having support to consolidate the new graduate's skill base}

Many of the managers reported putting a plan in place to assist the new graduates to consolidate their skills base, for example, "we have a plan and I sit down with them I would like you to achieve this in one month" (no 14) and "setting up almost like a plan of you know what do I need to achieve, what are my goals, how I am going to get there" (no 13). Some reported formalised processes, with another saying, "we did a skills inventory before and after three months and then at six months" (no 5).

There were different perspectives as to the readiness of new graduates for an independent role. One manager was completely confident the new graduates were ready for providing continuity of midwifery care for women through pregnancy birth and the postnatal period, "they are registered to practice midwifery to the full scope of practice" and "they have the skills and attributes to in fact do that" (no 2). Another said, "you have got to have confidence that they've got a certain level of skill" (no 6). This educator expressed another example of confidence in the new graduates "new grads come in knowing what they don't know, they know how to ask for help" (no 11). Conversely, another manager was more skeptical "when you're newly graduated, you actually don't even know what you don't know" (no 8).

\section{Finding support through team meetings}


Almost all the managers discussed team meetings as a form of support for new graduate midwives, illustrated by, "we have a meeting every Wednesday, we say you need to be part of that so they come to the meeting" (no 7) and the "teams meet each week with any concerns or questions" (no 1). Another said, "[we have] weekly meetings where they would all bring food and have case reviews and talk about what they did, what helped and what didn't help" (no 5). The managers discussed their role in supporting the new graduate through regular meetings explaining, "they officially/unofficially meet up with me on a weekly basis and then on a monthly basis" (no 14) and "they do feel supported we meet with them quite regularly in their first you know couple of months to see how they're tracking and how its working for them" (no 1).

\section{Being supported by the senior management}

The managers looked for support to employ new graduate midwives from the higher-level management. Two managers discussed the support they received from senior management saying "the overarching manager was very flexible" (no 5) and "the directors of midwifery we had were very committed to supporting midwives to work in this model" (no 1). Another said, "even our senior nurse manager, our senior operations manager, they know that if they [new graduates working in continuity models] have issues that can't be resolved by any of us they can go to them" (no 12). When the senior management was supportive of new graduates working in midwifery continuity of care models they were viewed as visionary, for example "it is visionary leadership who understand maternity services" and "it was the leadership we had supporting university teaching, being a practitioner in your own right" (no5).

Some managers discussed needing to convince their senior management that new graduates working in continuity of care models was safe, for example, "I suppose we have to get midwifery leadership group to recognise that the students who become registered are registered" (no 2). The manager was referring to the new graduate meeting the competency standards of the midwife and being a safe practitioner. There was some discussion from managers when new graduates worked in a standard transitional program they lost their ability 
to provide care across the scope of practice as described here, "the new leader has talked about new grads going into caseload because she doesn't want them tainted" (no 5). This manager discussed convincing senior management of the maternity service saying "you need to sell your successes" (no 2).

In some places, that senior management was not supportive as stated here, "I wonder if management just thinks it's all too hard" (no11). There was an optimistic outlook from this manager, "we have got some better team leaders in our newer staff than in our older staff by years of experience" and "the way of changing forwards, through midwifery leadership [visionary leadership] is with our new graduates" (no 14).

\section{Barriers - Managing the myths}

'We need to manage the fear around employing new graduates into continuity of care models"

There were concerns about an increase in adverse events, if new graduates were working in midwifery continuity of care models, with one manager saying "managers and executives fear that something bad is going to happen to a woman or a baby" (no1) and another "it is mostly managers who are the most nervous" (no 4). There was a thread of fear coming from senior management for example "they are nervous, they are frightened they don't have enough skilled leaders who are confident in supporting midwives to be self-determining in the workforce" (no 4). Maternity services and obstetrics is a highly litigious environment as reported by this manager, "the heads of department are all looking at medico legal issues" (no 15). It was difficult for this group of managers to advocate for new graduates when the management were medicolegally defensive about previous adverse events, for example "the midwifery managers as a group decide who works where and what skills people have and I guess for them they are looking at what IIMS [incident reporting system for adverse outcomes] are going to be in and what they felt are going to be the complaints" (no 15).

Fear also came from the new graduate's colleagues, the core staff with one saying "the older midwives would say they felt nervous for them" (no 5). Some 
reported the new graduate's being fearful, illustrated by this quote "one new graduate only lasted 6 weeks, I think it was her personal fear of working autonomously" (no 15) and discussed here, "you really have to want to do it and not be frightened of it" (no 7). On the other hand one manager stated, "because they have an expectation of learning they don't have a fear of it" (no 14).

\section{Changing organisational culture}

\section{"Culture is a challenge not a barrier"}

Managers discussed the need for new graduates to gain experience before working in continuity of care models based on history with one saying, "I think we still have a culture where if you don't do your penance in the birth suite, then you're not experienced enough to care for women independently" (no 10). Illustrated by this quote, "sort of a backlash from senior people [midwives], we still have a lot of people [core midwifery staff] who think you have to be in a place for a long time before you should be a caseload midwife" (no11) and here "it's the nature of working in a hospital, you know it's based on history" and "this is how we have always done things" (no 2). Finally, this manager challenged the historical nature of organisational culture saying "once you start having these rules around who can come to you, you do look a bit exclusive it's a bit crazy because it's a midwifery model" (no7).

There were reports of attempting to change the culture "to change the culture, the perception of staff on what a midwife is and what a midwife can do" (no 10). One manager reported attempting to change the organisational culture, trying to "use the workforce in a different way" and "use your staff in a slightly better or different way" (no 2). However, many of the managers were complacent about the culture, saying "it is the whole service and the culture around it is very medicalised" (no 4). Put simply by this manager "significant culture issues in our maternity unit anyway" meaning, the culture is medicalised and not very midwifery focused and it is "the general culture of the organisation" (no 1).

\section{Discussion}


This study provides important insights into the role of managers in new models of midwifery care, and provides key learnings for all managers in a changing health care environment. This study found that staffing the midwifery models was the main driver for employing newly graduated midwives into the continuity of care models. Managing a feminised workforce that has potential for frequent episodes of maternity leave with limited capacity for part time work (Forster et al. 2011, Hartz et al. 2012) has meant that managers need new graduate midwives to staff their continuity of care models. Building workforce capacity was seen as enabling the employment of new graduate midwives into the midwifery continuity of care models. Some units in Australia have undertaken workforce capacity building through clinical redesign. For example, one large tertiary referral hospital in metropolitan Sydney undertook a well-planned clinical redesign when introducing large numbers of midwives to work in continuity of care models (MGP), one of the strategies for sustaining the models included the recruitment of new graduate midwives to work in the MGP (Hartz et al. 2012).

Exposing students to midwifery continuity of care during their undergraduate programs was found to be important in our study, to enable the employment of new graduate midwives into continuity of care models upon graduation. This important finding from the managers and other key stakeholders, supports our previous findings and that of others, new graduate midwives felt prepared to work in midwifery continuity by completing their degree (Gray et al. 2012, Cummins et al. 2015). Without this early experience, retention of midwives may be at risk due to dissatisfaction with working in a standard rotation model when new graduate midwives have had experience in midwifery continuity of care model.

The initial support from the managers and senior management was found to be an enabler to employing new graduate midwives in midwifery continuity of care models. The initial support included a prolonged orientation period with a reduced caseload. Similarly, a Canadian study found one of the most positive experiences for new graduate nurses was a long ( $>4$ weeks) orientation period (Rush, et al. 2015). The other initial support finding, in our study, was a reduced 
workload with new graduate midwives having a lesser caseload initially. High workload, described as nurse/patient ratio, has been discussed as detrimental to the new graduate nurses' experience of their first year of practice (Feng and Tsai 2012). Other studies of new graduate nurses have found that job satisfaction, professional competence and turnover rates are related to adequate staffing (Numminen et al. 2015, Pineau et al. 2015). Therefore, the findings in our study of offering new graduate midwives a long orientation period and initially a reduced caseload/workload may enable the employment and retention of new graduate midwives.

In our study, support from the hospital senior management was integral in employing new graduate midwives into midwifery continuity of care models. It has been reported that the sustainability of midwifery continuity of care models depends on senior management to meet regularly and complete continuous assessment of the model (Hartz et al. 2012) and further that caseload midwifery would only be normalised as part of the maternity services with support from management (Forster et al. 2011). Hence visionary leadership is a critical driver and enabler to employing new graduate midwives to work in midwifery continuity of care models.

We found there were only a few barriers to employing new graduate midwives into midwifery continuity of care models. The barriers focused on safety and fears that something was going to happen to women and babies. The managers were able to manage the fears expressed from higher management despite a thread of nervousness from the core midwifery staff. It has been found that stand-alone midwifery led units in England are more successful when there is collegiality between all the midwifery and medical team and supportive management (Rayment et al. 2015). Despite the fears, the managers were focused on ensuring the new graduate midwives built their skills within the continuity of care models and over time they were confident to provide care to women across the full scope of midwifery practice.

\section{Limitations}


This study is limited to the views of Australian midwifery continuity of care managers and stakeholders.

\section{Conclusions}

We found a key driver for the employment of new graduate midwives to work in midwifery continuity of care models was the need to staff the models of care. The recruitment and retention of new graduate nurses has been examined in the literature as dependent upon work environment, collegial relationships and job satisfaction (Feng \& Tsai 2012; Laschinger 2012). We have found in this study that managers providing a longer orientation period are attracting new graduates to work in the continuity of care models. Collegiality is evident through the team meetings that the managers and other key stakeholders often attend and acts as a facilitator to retaining new graduate midwives working in the continuity of care model. Ongoing support is also important and new graduates midwives in New Zealand are mentored into midwifery continuity of care models (Lennox \& Foureur 2012).

\section{Implications for management}

Consequently, managers who provide a supportive orientation period with an initial reduced workload and ongoing support will attract and retain midwives to work in the midwifery continuity of care models. Despite these interventions and confidence that new graduates are competent to work across the scope of practice the managers are challenged by organisational culture. Visionary leadership and managing myths that mothers and babies may be at risk can be seen as critical to breaking down the barriers to employing new graduates into midwifery continuity of care models.

Further research is needed to discover why there are larger organisational challenges to employing new graduate midwives to work in midwifery continuity of care models. 


\section{REFERENCES}

Australian Government Department of Health and Ageing (2009). Maternity Services Reform, Deptartment of Health and Aging. Canberra, Commonwealth of Australia.

Barry, M. (2011 ). Newly-graduated Midwives Applying the Philosophy of Midwifery: A Theory of Transcending Barriers 2011 A Midwifery Odyssey Sydney

Burns, E., Fenwick, J., Schmied, V. \& Sheehan, A. 2012, 'Reflexivity in midwifery reserach: the insider/outsider debate ', Midwifery vol. 28, pp. 52-60.

Collins, C. T., J. Fereday, J. Pincombe, C. Oster and D. Turnbull (2010). "An evaluation of the satisfaction of midwives' working in midwifery group practice." Midwifery 26(4): 435-441

Cummins, A. M., E. Denney-Wilson and C. S. E. Homer (2015). "The experiences of new graduate midwives working in midwifery continuity of care models in Australia." Midwifery 31 (4).

Davies, S., Mason J., (2009). "Preceptorship for newly-qualified midwives: time for a change? ." British Journal of Midwifery 17(12): 804-805.

Davis, D., M. Foureur, V. Clements, P. Brodie and P. Herbison (2011). "The self reported confidence of newly graduated midwives before and after their first year of practice in Sydney, Australia " Women and Birth.

Feng, R. F. and Y. F. Tsai (2012). "Socialisation of new graduate nurses to practising nurses." Journal of clinical nursing 21(13 - 14): 2064-2071.

Forster, D. A., M. Newton, H. L. McLachlan and K. Willis (2011). "Exploring implementation and sustainability of models of care: can theory help?" BMC Public Health 11(Suppl 5): S8

Gray, J. E., N. Leap, A. Sheehy and C. S. E. Homer (2012). "The 'followthrough' experience in three-year Bachelor of Midwifery programs in Australia: A survey of students." Nurse Education in Practice 12(5): 258-263.

Hartz, D. L., J. White, K. A. Lainchbury, H. Gunn, H. Jarman, A. W. Welsh, D. Challis and S. K. Tracy (2012). "Australian maternity reform through clinical redesign." Australian Health Review 36(2): 6-6.

Holt, A. 2010, 'Using the telephone for narrative interviewing: a research note', Qualitative Research, vol. 10, no. 1, pp. 113-21. 
Hughes, A. and D. Fraser (2011). "'SINK or SWIM': The experience of newly qualified midwives in England." Midwifery 27: 382-386.

Kensington, M. (2006). "The faces of mentoring in New Zealand: Realities for the new graduate midwife " New Zealand College of Midwives Journal 35(oct).

Laschinger, H.K.S. 2012, 'Job and career satisfaction and turnover intentions of newly graduated nurses', Journal of nursing management, vol. 20, no. 4, pp. 472-84.

Lavender, T., Edwards, G. \& Alfirevic, Z. 2004, Demystifying Qualitative Reserach in Pregnancy and Childbirth Quay books MA Healthcare Ltd Wiltshire.

Lennox, S. \& Foureur, M. 2012, 'Developmental mentoring: New graduates' confidence grows when their needs shape the relationship ', New Zealand Com Journal

Lennox, S., A. Jutel and M. Foureur (2012). "The concerns of competent novices during a mentoring year." Nursing research and practice 2012.

Lennox, S., J. Skinner and M. Foureur (2008). "Mentorship preceptorship and clinical supervision: Three key processes for supporting midwives " Zealand College of Midwives Journal 39: 7-12.

McLachlan, H. L., D. A. Forster, M. A. Davey, T. Farrell, L. Gold, M. A. Biro, L. Albers, M. Flood, J. Oats and U. Waldenstro (2012). "Effects of continuity of care by a primary midwife (caseload midwifery) on caesarean section rates in women of low obstetric risk: the COSMOS randomised controlled trial." British Journal of Obstetrics and Gynaecology

New South Wales Health (2010). Maternity - Towards normal birth N. S. W. Ministry of. Health. North Sydney New South Wales Health

Newton, M. S., H. L. McLachlan, K. F. Willis and D. A. Forster (2014). "Are caseload midwives more satisfied and less burnt out than standard care midwives? Findings from two cross-sectional surveys conducted in Victoria, Australia." BMC pregnancy and childbirth 14: 426.

Numminen, O., E. Ruoppa, H. Leino - Kilpi, H. Isoaho, M. Hupli and R. Meretoja (2015). "Practice environment and its association with professional competence and work - related factors: perception of newly graduated nurses." Journal of nursing management. 
Panettiere, M. and T. Cadman (2002). "Do new graduate midwives need extra support? Are direct-entry midwifery graduates adequately prepared to practise in the hospital environment? A clinical midwifery leader and a nurse educator put the case for an entry-to-practice programme for midwives.(viewpoint)." Kai Tiaki: Nursing New Zealand 8(3): 18(12).

Passant, L., C. S. E. Homer and J. Wills (2003). "From student to midwife: the experiences of newly graduated midwives working in an innovative model of midwifery care." Australian Midwifery 16(4): 18-21.

Pineau Stam, L. M., H. K. Spence Laschinger, S. Regan and C. A. Wong (2015). "The influence of personal and workplace resources on new graduate nurses' job satisfaction." Journal of nursing management 23(2): 190-199.

Rayment, J., C. McCourt, S. Rance and J. Sandall (2015). "What makes alongside midwifery-led units work? Lessons from a national research project." The Practising Midwife 18(6): 31-33.

Rush, K. L., M. Adamack, J. Gordon, R. Janke and I. R. Ghement (2015). "Orientation and transition programme component predictors of new graduate workplace integration." Journal of nursing management 23(2): 143-155.

Sandall, J., H. Soltani, S. Gates, A. Shennan and D. Devane (2013). "Midwifeled continuity models versus other models of care for childbearing women." Cochrane database of systematic reviews(8).

Sandelowski, M. 2000 'Whatever happened to qualitative description? ', Research in Nursing and Health vol. 23, pp. 334-40.

Tracy, S. K., D. L. Hartz, M. B. Tracy, J. Allen, A. Forti, B. Hall, J. White, A. Lainchbury, H. Stapleton, M. Beckmann, A. Bisits, C. Homer, M. Foureur, A. Welsh and S. Kildea (2013) "Caseload midwifery care versus standard maternity care for women of any risk: M@NGO, a randomised controlled trial." The Lancet 382(9906): 1723-1732.

Ward, K., Gott, M. \& Hoare, K. 2015, 'Participants' views of telephone interviews within a grounded theory study', Journal of Advanced Nursing, pp. n/a-n/a. 
Figure 1: Model of visionary leadership

Having visionary leadership to enable innovation

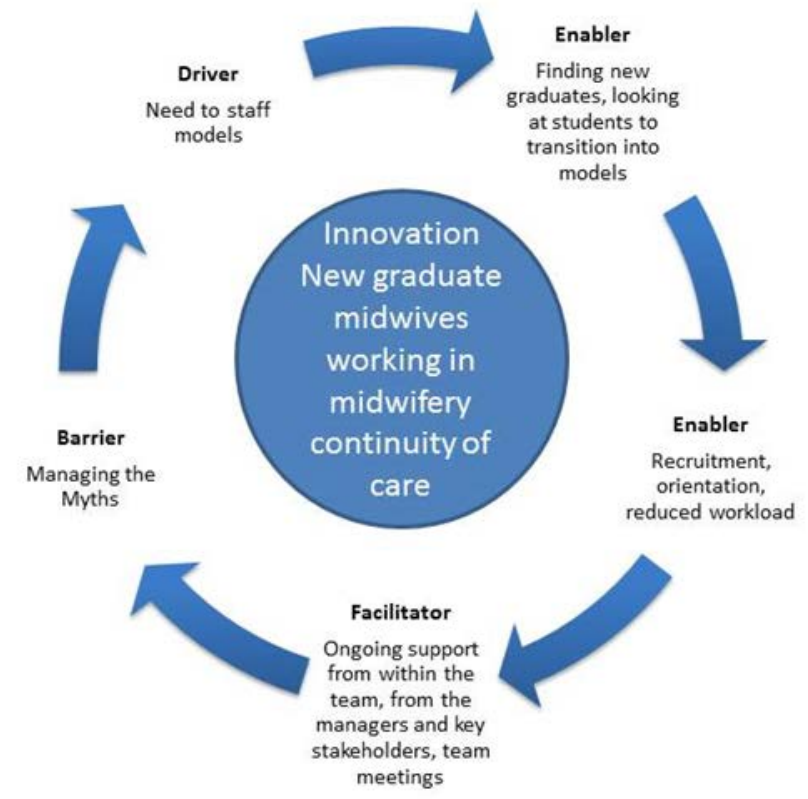


Table 1 - Questions used in the interviews

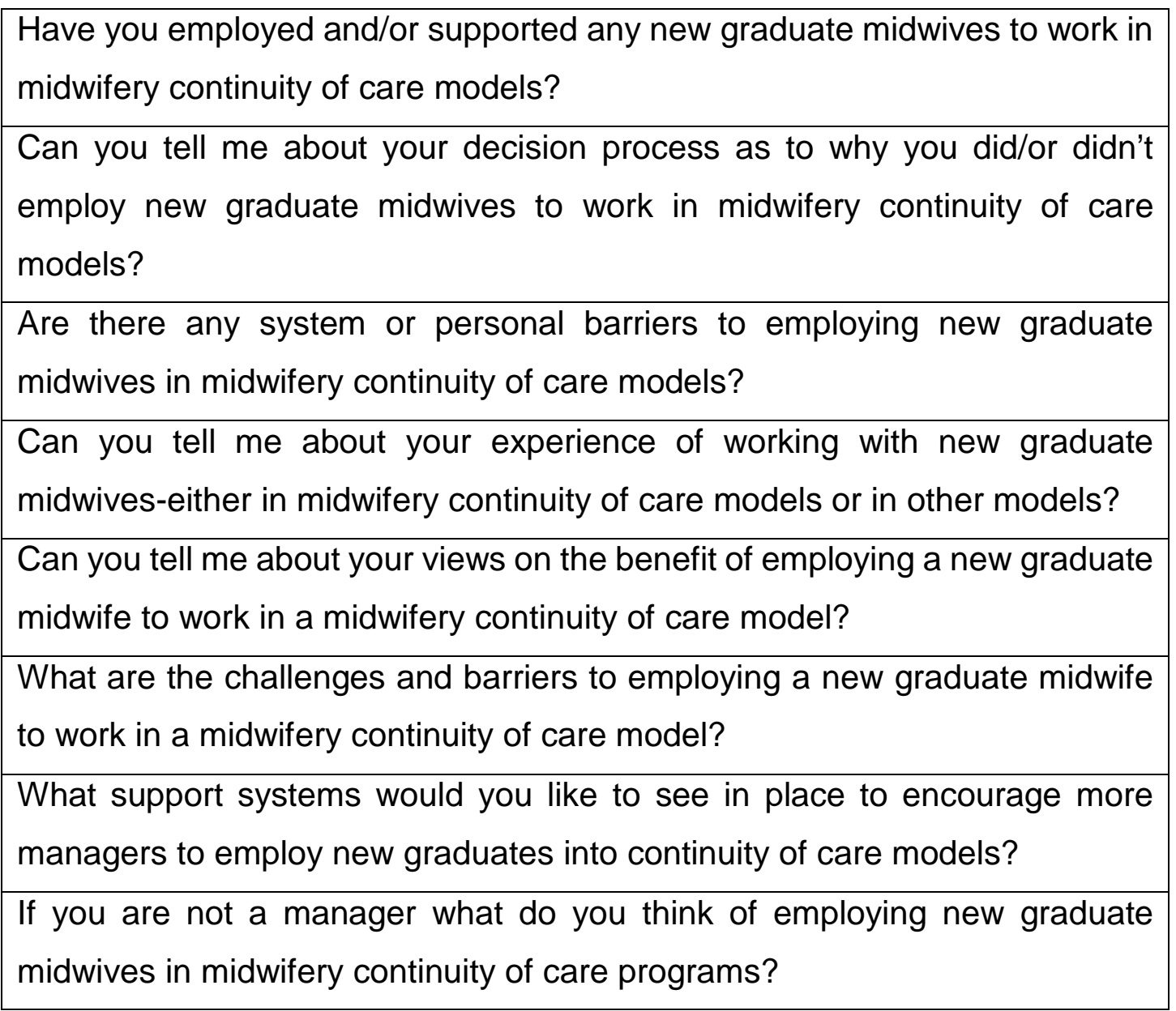

